\title{
Power Adaptation for Multihop Networks With End-to-End BER Requirements
}

\author{
Hongbing Cheng, Member, IEEE, and Yu-Dong Yao, Senior Member, IEEE
}

\begin{abstract}
With the requirement of average end-to-end bit error rate (BER) performance, we study power-adaptation problems for multihop ad hoc networks to minimize the power consumption in fading channels, considering two scenarios with regard to the knowledge of the channel-state information (CSI). One is that the transmitter for each hop knows the instantaneous CSI (ICSI) of its hop and all other hops (ICSI/ICSI), and the other is that the transmitter of each hop has only the knowledge of the ICSI of its hop and the average CSI (ACSI) of all other hops (ICSI/ACSI). The two power-adaptation problems (with ICSI/ICSI and ICSI/ACSI) are formulated into nonlinear programming problems, and corresponding power-adaptation solutions/schemes are obtained. $\mathrm{Nu}$ merical examples are presented to compare the networks with the two proposed power-adaptation schemes and a distributed poweradaptation scheme, in which the transmitter of each individual hop adjusts its transmission power based on its own ICSI and a specified BER requirement for this hop. It is shown that the two proposed schemes have similar power consumptions, and both achieve a noticeable power saving over the distributed scheme.
\end{abstract}

Index Terms-End-to-end bit error rate (BER), multihop networks, optimization, power adaptation.

\section{INTRODUCTION}

I $\mathrm{N}$ MANY ad hoc networks, including sensor networks and battlefield networks, energy is a very limited resource, because most communication nodes are battery operated, and replacing these batteries can be difficult. Therefore, the issue of energy or power minimization has been investigated by many researchers at different layers of the protocol stack, including modulation and power adaptation in the physical layer [1], medium access control (MAC) protocol design in the MAC layer [2], and routing-scheme design in the network layer [3]. Since all layers of the protocol stack affect the energy consumption of data transmissions, the cross-layer design method is also explored for the energy minimization [4], [5].

The multihop relay transmission is also an effective approach for energy or power saving in ad hoc networks [6], [7]. It routes information bits through relay nodes to reduce the transmission power of each node. The capacity and outage performance of multihop relay networks with or without cooperative diversity are well studied [8], [9]. In addition, the power-adaptation issue for multihop networks has been studied by many researchers

Manuscript received October 4, 2009; revised May 25, 2010; accepted June 3, 2010. Date of publication June 14, 2010; date of current version September 17, 2010. The review of this paper was coordinated by Prof. H.-F. Lu.

The authors are with the Wireless Information System Engineering Laboratory, Department of Electrical and Computer Engineering, Stevens Institute of Technology, Hoboken, NJ 07030 USA (e-mail: hcheng@ @stevens.edu; yyao@stevens.edu).

Color versions of one or more of the figures in this paper are available online at http://ieeexplore.ieee.org.

Digital Object Identifier 10.1109/TVT.2010.2053057 from an information-theoretic point of view, where the achieved data rate or outage probability is used to quantify the link quality [10]-[12]. Some other researchers studied the power adaptation to maximize the signal-to-noise ratio (SNR) for multihop relay networks [13]. In a practical communication system, a target bit error rate (BER) or symbol error rate (SER) is usually given as a requirement of the link quality in the physical layer. There are two approaches to specify a BER requirement for a multihop link: One is an individual-hop BER requirement, and the other is an end-to-end (from source to destination) BER requirement. The investigation of power adaptation in a multihop network with the individual-hop BER requirement is equivalent to the consideration of a single-hop network, which has extensively been studied [14]-[16]. The energy-minimized power adaptation with the constraint of the end-to-end BER requirement is optimized in [7] for a multihop network with binary phase-shift keying (BPSK) modulation, and the result is compared with the case with the individualhop BER requirement. It assumes that only the knowledge of the average channel-state information (ACSI) is available at the transmitter and the relay nodes. A power-adaptation problem with the instantaneous CSI (ICSI) is investigated in [17] to minimize the average end-to-end decoded SER with a constraint of average power consumption. The optimizations in both [7] and [17] only obtain closed-form solutions for BPSK modulation, even with approximations. By approximating the instantaneous end-to-end SER with an upper bound, Zhang and Gong [18] extended the work in [17] and studied the power-adaptation issue in multihop relay networks for any given modulation format, with the objective of minimizing the instantaneous SER and a constraint of the instantaneous transmit power. However, the average power consumption and the average SER or BER performance are not considered.

In this paper, we study power adaptation issues in multihop networks to minimize the average power consumption with a given requirement of the average end-to-end BER performance. Specifically, two different scenarios with regard to the knowledge of the CSI are considered. One is that the transmitter for each hop knows the ICSI of this hop and all other hops (ICSI/ICSI), and the other is that the transmitter of each hop has only the knowledge of the ICSI of its hop and the ACSI of all other hops (ICSI/ACSI). Necessary conditions for the optimal power adaptation are summarized for general multihop networks, and closed-form approximations of the optimal power-adaptation solutions are derived for multihop networks with multilevel quadrature amplitude modulation (MQAM) or multiple phase-shift keying (MPSK) modulations. Numerical results are presented to show the power saving (as compared 
with the optimization based on the individual-hop BER requirement) achieved through the proposed optimizations.

The remainder of this paper is organized as follows: In Section II, we describe the system model and derive the endto-end BER expression. In Sections III and IV, the poweradaptation problems in the two scenarios with different CSI knowledge are modeled and solved, respectively. Section V gives some numerical results, and Section VI summarizes our conclusions.

\section{SySTEM MODEL}

\section{A. Network Model}

We consider a regenerative multihop relay network, where a source node communicates with a destination node through $N-1$ relay nodes. The routing issue is determined by upper layers and is not considered in our work. When a relay node receives the information bits from the source node or its preceding relay node, it first detects and then remodulates for transmission to the next relay node or the destination node. We assume that there is no error detection at the relay nodes, following the detection and forward strategy modeled in [7] and [19].

We denote the source node as node 0 , the destination node as node $N$, and the relay nodes as node 1 to $N-1$. The communication link from node $(i-1)$ to node $i$ is called the $i$ th hop $(1 \leq i \leq N)$. The transmission power allocated to the $i$ th hop is $P_{t i}$, which may vary according to the channel states. The same modulation format is used for each hop and is constant for different channel states. For the transmission from node $j(0 \leq j \leq N-1)$ to node $i$, the BER is assumed to be $p_{j, i}$, and the BER of the $i$ th hop is $p_{i}=p_{i-1, i}$.

With the preceding network model, the average power consumption of a multihop network is

$$
\bar{P}_{t}=\mathbf{E}\left[\sum_{i=1}^{N} P_{t i}\right]
$$

where $\mathbf{E}[$.$] denotes the expectation over different channel states.$

\section{B. End-to-End BER Derivations}

For a bit transmitted from node 0 to node $i$, an error event occurs at node $i$ in the following two situations only: 1) The bit is in error at node $(i-1)$, and the transmission through hop $i$ (from node $(i-1)$ to node $i$ ) is correct. 2) The bit is correct at node $(i-1)$, and the transmission through hop $i$ is incorrect. Therefore, if the BER of each hop is independent, the BER from node 0 to node $i$ can recursively be calculated as

$$
\begin{aligned}
p_{0, i} & =p_{0, i-1}\left(1-p_{i}\right)+p_{i}\left(1-p_{0, i-1}\right) \\
& =\frac{1-\left(1-2 p_{0, i-1}\right)\left(1-2 p_{i}\right)}{2}
\end{aligned}
$$

With the use of (2), we can get a simple closed-form expression of the end-to-end BER for a link with $N$ independent hops

$$
p_{0, N}=\frac{1-\prod_{i=1}^{N}\left(1-2 p_{i}\right)}{2} .
$$

The derivation of (3) is given in Appendix A. This expression of BER is equivalent to that derived in [19], but it has a simpler structure and will facilitate our optimization work in the next section.

\section{Channel Model}

We consider a block-fading channel model, i.e., the channel fading is constant within a data block and independently varies in different blocks [20]. The length of the data block is assumed to be $L$ bits. Considering the large-scale path loss and the smallscale Rayleigh fading, the instantaneous SNR of a data block at the receiver of the $i$ th hop is

$$
\gamma_{i}=\frac{G_{i} \alpha_{i} P_{t i}}{N_{0}}=\tilde{\gamma}_{i} P_{t i}
$$

for $i=1,2, \ldots, N$, where $\tilde{\gamma}_{i}=G_{i} \alpha_{i} / N_{0}$ is a normalized SNR; $G_{i}=G_{0} r_{i}^{-n}$ is the path loss; $G_{0}$ is a reference power gain factor at a communication distance of $1 \mathrm{~m} ; r_{i}$ and $P_{t i}$ are the communication distance and transmit power of the $i$ th hop, respectively; $n$ is the path loss factor; $N_{0}$ is the noise power; and $\alpha_{i}$ is the instantaneous channel power gain factor, whose probability density function (pdf) is

$$
f\left(\alpha_{i}\right)= \begin{cases}e^{-\alpha_{i}}, & \alpha_{i} \geq 0 \\ 0, & \alpha_{i}<0 .\end{cases}
$$

The instantaneous BER of each hop is a decreasing function of its instantaneous SNR and is denoted by $p_{e}\left(\gamma_{i}\right)$ for $i=$ $1,2, \ldots, N$.

\section{Problem Formulation}

The objective of this research is to optimize the power allocation for each hop in a multihop network to minimize the overall power consumption per bit transmission with a specified average end-to-end BER requirement of $p_{\text {req. }}$. With the aforementioned network and channel models, the power minimization optimization problem is modeled as

$$
\begin{array}{cl}
\underset{\left\{P_{t 1}, P_{t 2}, \ldots, P_{t N}\right\}}{\min } & \bar{P}_{t} \\
\text { s.t. } & \bar{p}_{0, N} \leq p_{\text {req }} \\
& P_{t i} \geq 0, i=1,2, \ldots, N
\end{array}
$$

where $\bar{p}_{0, N}$ is the average end-to-end BER, and $\bar{p}_{0, N}=$ $\mathbf{E}\left[p_{0, N}\right]$.

Notice that, although the rate requirement is not explicitly stated in the preceding optimization problem, an implicit requirement of a constant data rate is considered in our network model since a fixed modulation format is assumed for each hop in any channel state (unconditional transmission [21]). In other words, each node will transmit with a constant data rate in different channel states, no matter how much transmit power is allocated (including zero transmit power) [21], [22].

\section{Power Adaptation FOR The ICSI/ICSI SCEnARIO}

We first consider the power adaptation in a scenario in which each node has the knowledge of the ICSI of not only its next 
of hop but all other hops as well, as in [11] and [12]. We refer to this scenario as ICSI/ICSI in the following. It can be used to represent multihop networks in which the channel fading is slow and the receiver of each hop can estimate the ICSI and send it to all other nodes. Although the assumption of ICSI/ICSI is ideal, this will provide a lower bound for the power consumption in multihop networks with power adaptation. In this scenario, the transmission power allocated to each node is a function of the normalized SNR of all the hops; therefore, we denote the instantaneous transmit power as $P_{t i}(\tilde{\boldsymbol{\Gamma}})$, where $\tilde{\boldsymbol{\Gamma}}=\left(\tilde{\gamma}_{1}, \tilde{\gamma}_{2}, \ldots, \tilde{\gamma}_{N}\right)$. The average power consumption in the network is thus calculated as

$$
\bar{P}_{t}=\int_{\mathcal{A} \in R_{+}^{N}}\left[\sum_{i=1}^{N} P_{t i}(\tilde{\boldsymbol{\Gamma}})\right] \tilde{f}(\mathcal{A}) d \mathcal{A}
$$

where $\mathcal{A}$ is the channel gain vector defined by $\mathcal{A}=$ $\left(\alpha_{1}, \alpha_{2}, \ldots, \alpha_{N}\right), \tilde{f}(\mathcal{A})=\prod_{i=1}^{N} f\left(\alpha_{i}\right)$ is the pdf of $\mathcal{A}$, and $R_{+}^{N}$ denotes the $N$-dimensional nonnegative real Euclidean space, which is composed of all nonnegative $N$-dimensional real vectors.

Notice that the average BER of each hop is not independent since the transmission power of each node relates to the ICSI of all hops. Therefore, (3) cannot directly be used to calculate the average end-to-end BER. However, for each given value of $\mathcal{A}$, the channels of all the hops can be regarded as cascaded independent Gaussian channels, where (3) applies. The average end-to-end BER is then calculated as

$$
\bar{p}_{0, N}=\int_{\mathcal{A} \in R_{+}^{N}} \frac{1-\prod_{i=1}^{N}\left[1-2 p_{e}\left(\gamma_{i}\right)\right]}{2} \tilde{f}(\mathcal{A}) d \mathcal{A} .
$$

\section{A. General Case}

By substituting (7) and (8) into (6), the power minimization problem with ICSI/ICSI is given. A necessary condition of the optimal solution to this problem for any form of the BER function $p_{e}\left(\gamma_{i}\right)$ (for different modulation schemes) is given as follows:

Theorem 1: In the ICSI/ICSI scenario, the optimal transmit power allocated to the $i$ th hop $P_{t i}^{*}(\tilde{\boldsymbol{\Gamma}})$ is a function of all hops' channel gains and satisfies $P_{t i}^{*}(\tilde{\boldsymbol{\Gamma}})=0$, or

$$
\frac{p_{s}\left(\tilde{\gamma}_{i} P_{t i}^{*}(\tilde{\boldsymbol{\Gamma}})\right)}{p_{s}^{\prime}\left(\tilde{\gamma}_{i} P_{t i}^{*}(\tilde{\boldsymbol{\Gamma}})\right)}=\frac{1}{2} \lambda \tilde{\gamma}_{i} \rho(\tilde{\boldsymbol{\Gamma}}), \quad\left(P_{t i}^{*}(\tilde{\boldsymbol{\Gamma}})>0\right)
$$

for $i=1,2, \ldots, N$, where $\lambda$ is a positive constant, and $\rho(\tilde{\boldsymbol{\Gamma}})=$ $\prod_{i=1}^{N} p_{s}\left(\tilde{\gamma}_{i} P_{t i}^{*}(\tilde{\boldsymbol{\Gamma}})\right) \cdot p_{s}\left(\gamma_{i}\right)$ is a function of $\gamma_{i}$ defined as $p_{s}\left(\gamma_{i}\right)=1-2 p_{e}\left(\gamma_{i}\right)$, and $p_{s}^{\prime}\left(\gamma_{i}\right)$ is its first-order derivative. The average value of $\rho(\tilde{\boldsymbol{\Gamma}})$ over different fading states satisfies

$$
\bar{\rho}=\int_{\mathcal{A} \in R_{+}^{N}} \rho(\tilde{\boldsymbol{\Gamma}}) \tilde{f}(\mathcal{A}) d \mathcal{A}=1-2 p_{\text {req }} .
$$

The proof of the theorem is shown in Appendix B. Now, we focus on the derivation of the optimal power allocation for some specific modulation schemes according to the preceding theorem. For notational convenience, we omit the explicit dependence on $\tilde{\boldsymbol{\Gamma}}$ and denote $P_{t i}^{*}(\tilde{\boldsymbol{\Gamma}})$ as $P_{t i}^{*}$ and $\rho(\tilde{\boldsymbol{\Gamma}})$ as $\rho$ in the following derivations.

\section{B. MQAM or MPSK Modulations}

It is shown in [16] that the instantaneous BER of the MQAM and MPSK modulations can be approximated by

$$
p_{e}\left(\gamma_{i}\right)=a e^{-b \gamma_{i}}
$$

where $a$ and $b$ are determined by the modulation format and order, and $a<0.5$. With this approximation and the definition of $p_{s}\left(\gamma_{i}\right)$

$$
p_{s}^{\prime}\left(\gamma_{i}\right)=b\left[1-p_{s}\left(\gamma_{i}\right)\right] .
$$

By inserting (12) into (9)

$$
p_{s}\left(\tilde{\gamma}_{i} P_{t i}^{*}\right)=\frac{\tilde{\lambda} \tilde{\gamma}_{i} \rho}{1+\tilde{\lambda} \tilde{\gamma}_{i} \rho}
$$

where $\tilde{\lambda}=(1 / 2) b \lambda$. Therefore, the possible optimal transmit power of the $i$ th hop is $P_{t i}^{*}=0$, or

$$
P_{t i}^{*}=\frac{1}{b \tilde{\gamma}_{i}} \ln \left[2 a\left(1+\tilde{\lambda} \tilde{\gamma}_{i} \rho\right)\right] .
$$

According to its definition, $\rho$ is a solution of the following equation:

$$
\prod_{i=1}^{N} \frac{\tilde{\lambda} \tilde{\gamma}_{i} \rho}{1+\tilde{\lambda} \tilde{\gamma}_{i} \rho}=\rho
$$

for any given $\tilde{\gamma}_{i}, i=1,2, \ldots, N$.

Before solving the preceding equation, we consider a special case where the equation has no solution in the region of $\rho \in$ $\left[\rho_{\min }, 1\right)$, where $\rho_{\min }=((1 / 2 a)-1)\left(1 / \tilde{\lambda} \tilde{\gamma}_{\min }\right)$, and $\tilde{\gamma}_{\min }=$ $\min \left(\tilde{\gamma}_{1}, \tilde{\gamma}_{2}, \ldots, \tilde{\gamma}_{N}\right)$. In this case, zero transmit power should be allocated to at least one hop in the link since it is the only possible optimum solution satisfying $P_{t i}^{*} \geq 0$, according to Theorem 1. Therefore, the link is disconnected, and the endto-end BER is 0.5 , no matter how large the powers allocated to other hops are. It is clear that we should set the transmit power of all hops to 0 for power saving in this case. Notice that the zero transmit power will not decrease the data rate since we assume that the unconditional transmission model is used and that the transmitter always transmits with a fixed modulation format, even when the allocated transmit power is equal to zero [21], [22]. However, if the transmit power is 0 , the receiver has to guess the information bits, and the end-to-end BER is always 0.5 . The average end-to-end BER in (8) is thus rewritten as

$$
\begin{aligned}
\bar{p}_{0, N}=0.5 \int_{\mathcal{A} \in \Omega} \tilde{f}(\mathcal{A}) d \mathcal{A} & \\
& +\int_{\mathcal{A} \in \bar{\Omega}} \frac{1-\prod_{i=1}^{N}\left[1-2 a e^{\left.-b \gamma_{i}\right]}\right.}{2} \tilde{f}(\mathcal{A}) d \mathcal{A}
\end{aligned}
$$

where $\Omega$ is the set of the channel states, which result in zero power allocation at each hop, and $\bar{\Omega}$ is its complement set. It 
is worth mentioning that, as seen in the preceding equation, although the instantaneous end-to-end BER is 0.5 when $\mathcal{A} \in \Omega$, the requirement of the average end-to-end BER in (6) can still be satisfied as long as the probability of $\mathcal{A} \in \Omega$ and the instantaneous end-to-end BER when $\mathcal{A} \in \bar{\Omega}$ are small enough. In general, the existence of the zero power allocation can also be understood as follows: In some bad channel states with small channel gains, a large transmit power helps little in the reduction of the instantaneous BER. However, in some good channel states with large channel gain, it may only consume a small amount of extra power to obtain the same reduction of instantaneous BER. Therefore, we set the transmit power to zero at the bad channel states to save power and allocate extra powers to good channel states so that the average end-to-end BER satisfies the requirement.

Now, we try to solve (15) and, first, consider a two-hop link, i.e., $N=2$. The equation is equivalent to a quadratic equation and has two positive solutions

$$
\begin{aligned}
\rho_{1,2}=\frac{1-\left(\frac{1}{\tilde{\gamma}_{1} \tilde{\lambda}}+\right.}{2} & \left.\frac{1}{\tilde{\gamma}_{2} \tilde{\lambda}}\right) \\
& \pm \frac{\sqrt{\left[1-\left(\frac{1}{\tilde{\gamma}_{1} \tilde{\lambda}}+\frac{1}{\tilde{\gamma}_{2} \tilde{\lambda}}\right)\right]^{2}-\frac{4}{\tilde{\gamma}_{1} \tilde{\gamma}_{2} \tilde{\lambda}^{2}}}}{2}
\end{aligned}
$$

when $\sqrt{\left(1 / \tilde{\gamma}_{1} \tilde{\lambda}\right)}+\sqrt{\left(1 / \tilde{\gamma}_{2} \tilde{\lambda}\right)} \leq 1$ and no positive solution otherwise. Since the larger solution $\rho_{1}$ satisfies that $\rho_{1} \geq$ $\sqrt{\left(1 / \tilde{\gamma}_{1} \tilde{\gamma}_{2} \tilde{\lambda}^{2}\right)}$, it is easy to validate that the optimization problem (6) is convex at the point of $P_{t i}=\left(1 / b \tilde{\gamma}_{i}\right) \ln [2 a(1+$ $\left.\left.\tilde{\lambda} \tilde{\gamma}_{i} \rho_{1}\right)\right], i=1,2$. Therefore, this point is the global optimal solution of the optimization problem (6) according to the Karush-Kuhn-Tucker (KKT) sufficient conditions [23]. The global optimal transmission power of a two-hop link is thus written as

$$
P_{t i}^{*}= \begin{cases}\frac{1}{b \tilde{\gamma}_{i}} \ln \left[2 a\left(1+\tilde{\lambda} \tilde{\gamma}_{i} \rho_{1}\right)\right], & \sqrt{\frac{1}{\tilde{\gamma}_{1} \tilde{\lambda}}}+\sqrt{\frac{1}{\tilde{\gamma}_{2} \tilde{\lambda}}} \leq 1 \\ 0, & \text { and } \rho_{1} \in\left[\rho_{\min }, 1\right) \\ & \text { otherwise }\end{cases}
$$

for $i=1,2$.

When the hop number $N>2$, it is hard to get a closedform solution for (15). An approximation solution is given as follows: First, by applying logarithm to both sides of (15), we transformed the equation to

$$
\sum_{i=1}^{N} \ln \left(1+\frac{1}{\tilde{\gamma}_{i} \tilde{\lambda} \rho}\right)=\ln \frac{1}{\rho} .
$$

Since $\bar{\rho}=1-2 p_{e}$ and the target end-to-end BER $p_{e}$ is much smaller than 1 , the value of $\rho$ should be much larger than one half; therefore, $\tilde{\lambda} \tilde{\gamma}_{i} \rho \gg 1$ under most fading conditions. We can thus approximate $\ln (1 / \rho) \approx 1-(1 / \rho)$ and $\ln \left(1+\left(1 / \tilde{\gamma}_{i} \tilde{\lambda} \rho\right)\right) \approx\left(1 / \tilde{\gamma}_{i} \tilde{\lambda} \rho\right)$ in $(19)$, according to the Taylor expansion, and obtain

$$
\rho \approx 1-\sum_{i=1}^{N} \frac{1}{\tilde{\gamma}_{i} \tilde{\lambda}}
$$

An approximation of the optimal power allocation for an $N$ hop link is thus obtained as

$$
\tilde{P}_{t i}^{*}= \begin{cases}\frac{1}{b \tilde{\gamma}_{i}} \ln \left[2 a\left(1+\tilde{\lambda} \tilde{\gamma}_{i} \tilde{\rho}\right],\right. & \tilde{\rho} \geq \rho_{\min } \\ 0, & \text { otherwise }\end{cases}
$$

for $i=1,2, \ldots, N$, where $\tilde{\rho}=1-\sum_{i=1}^{N}\left(1 / \tilde{\gamma}_{i} \tilde{\lambda}\right)$.

The constant $\tilde{\lambda}$ is a solution of (10), which can be obtained through a numerical search method for any fading channel model, as in [16]. With a given distribution of the small-scale channel fading, the value of $\tilde{\lambda}$ is only related to the ACSI of each hop.

If independent Rayleigh fading channels are assumed for all the hops, we are also able to obtain an approximation for $\tilde{\lambda}$ as

$$
\tilde{\lambda} \approx-N_{0} \frac{k_{1}}{2 p_{\text {req }}} W_{-1}\left(-\frac{2 p_{\text {req }} e^{-k_{2} / k_{1}}}{k_{1}}\right)
$$

where $k_{1}=\sum_{i=1}^{N}\left(1 / G_{i}\right)$, and $k_{2}=\sum_{i=1}^{N}\left(1 / G_{i}\right)((1 / 2 a)-$ $\left.\gamma-\ln \left(1 / 2 a G_{i}\right)\right) . \gamma$ is the Euler-Mascheroni constant [24]. $W_{-1}(x)$ is the branch of the Lambert $\mathrm{W}$ function that satisfies $W_{-1}(x) \leq-1$. The Lambert $\mathrm{W}$ function is defined as the multivalued inverse of the function $f(w)=w e^{w}$ [25]. The derivation of (22) is shown in Appendix C. The accuracy of this approximation will be examined in Section $\mathrm{V}$.

It is seen that the value of $\left(\tilde{\lambda} / N_{0}\right)$ is constant over different $N_{0}$ 's; therefore, both the power allocations in (18) and (21) are linearly increased with the noise power $N_{0}$. One can find that the power allocated for one hop in (21) approaches that for a single-hop link in [16] if the channel gains of other hops are much larger than that of this hop. Otherwise, the power of one hop will be affected by all hops' channels, particularly those worse than or closed to this hop's channel. In addition, the worst channel has the most impact. This is due to the fact that the hop with the worst channel dominates the end-to-end BER of a multihop link.

Notice that, in the preceding derivations, the distribution information of the channel gains is not required, except for the derivation of $\tilde{\lambda}$. Therefore, (21) is also valid for correlated fading channels, except that the value of $\tilde{\lambda}$ should be recalculated according to the joint pdf of the channel gains of all hops. Specifically, if the joint pdf of all hops' channel gains $f(\mathcal{A})$ is known at each node, we may insert it into (10) and then solve the equation to obtain the value of $\tilde{\lambda}$. However, it is hard to obtain a closed-form solution of $\tilde{\lambda}$.

\section{Extra Power Consumption to Obtain ICSI}

The power-adaptation scheme previously derived is based on the ideal assumption that each node has the ICSI of all hops. In reality, it consumes extra power to obtain such ICSI at each node, which impacts the power-saving performance of the proposed scheme [26]. In this section, we evaluate this extra power consumption with a simple ICSI transmission protocol, where each node periodically estimates the ICSI of its preceding hop (every data block, i.e., $L$ data bits) and then transmits the estimated ICSI to other nodes. Specifically, the $i$ th node estimated the ICSI of the $i$ th hop and transmits the ICSI 
to nodes $(i-1)$ and $(i+1)$. Then, node $(i-1)$ passes the ICSI to node $(i-2)$, node $(i+1)$ passes the ICSI to node $(i+2)$, and so on until all the nodes get the ICSI. Since the channel estimation is always performed for any communication link with coherent detection, we only consider the power consumption of the ICSI transmissions.

According to the preceding protocol, in an $N$-hop link, the ICSI of each hop, except the $N$ th hop, has to be transmitted $N-1$ times so that every node obtains the ICSI. In addition, the ICSI of the $N$ th hop has to be transmitted $N$ times. Therefore, the overall transmission times of the ICSI is $N^{2}-$ $N+1$. Assuming that the ICSI information contains $K$ bits, the increment percentage of the power consumption due to the ICSI transmission is

$$
\epsilon_{1}=\frac{\left(N^{2}-N+1\right) K}{L}
$$

In the conventional power-adaptation scheme for one-hop link, the receiver node only needs to feedback the ICSI to the transmitter node. Therefore, if we use the conventional singlehop power adaptation scheme to adjust the transmit power of each hop in an $N$-hop link, the ICSI only requires to be transmitted $N$ times. The increment percentage of the power consumption is

$$
\epsilon_{2}=\frac{N K}{L}
$$

It is seen that the power-consumption increment percentage of our proposed scheme $\left(\epsilon_{1}\right)$ is much larger than that of the conventional scheme $\left(\epsilon_{2}\right)$, particularly with large hop numbers. Therefore, the proposed power-adaptation scheme is more suitable for the systems with $K \ll L$, i.e., the channel fading slowly varies, or with a small hop number $N$.

\section{Power AdAPtation For The ICSI/ACSI SCEnARIO}

In this section, we consider the power adaptation in a more practical scenario, where each node has only the ICSI of the next hop and the ACSI of other hops, which is referred to as ICSI/ACSI. The receiver of each hop only needs to feed the estimated ICSI back to its own transmitter. Unlike the ICSI, the ACSI very slowly varies and remains constant in many data blocks since it is determined by the location of each node and, sometimes, shadow fading. Therefore, the overhead for the exchange of CSI at different nodes is significantly reduced [26]. The time and power consumptions in the exchange of ACSI are negligible, in comparison with those in the transmission of many data blocks following each ACSI update. Therefore, the extra power consumption to obtain ICSI in the ICSI/ACSI scenario is the same as that in the conventional single-hop power-adaptation scheme, as derived in (24).

In this scenario, the transmit power allocated to the $i$ th hop is a function of its own normalized SNR $\tilde{\gamma}_{i}$ and the average channel gain of other hops, and denoted as $P_{t i}\left(\tilde{\gamma}_{i}, \mathbf{G}_{\bar{i}}\right)$, where $\mathbf{G}_{\bar{i}}=\left(G_{1}, \ldots, G_{i-1}, G_{i+1}, \ldots, G_{N}\right)$. Therefore, the average power consumption and the average end-to-end BER are written as

$$
\begin{aligned}
\bar{P}_{t} & =\sum_{i=1}^{N} \int_{0}^{+\infty} P_{t i}\left(\tilde{\gamma}_{i}, \mathbf{G}_{\bar{i}}\right) f\left(\alpha_{i}\right) d \alpha_{i} \\
\bar{p}_{0, N} & =\frac{1-\prod_{i=1}^{N}\left[1-2 \bar{p}_{e, i}\right]}{2}
\end{aligned}
$$

where $\bar{p}_{e, i}=\int_{0}^{+\infty} p_{e}\left(\gamma_{i}\right) f\left(\alpha_{i}\right) d \alpha_{i}$. Similar to the preceding section, to simplify the notations, we will omit the explicit dependence of the power allocations on the channel states and denote $P_{t i}\left(\tilde{\gamma}_{i}, \mathbf{G}_{\bar{i}}\right)$ as $P_{t i}$ in the following derivations:

\section{A. General Case}

By inserting (25) and (26) into (6), the optimization problem with ICSI/ACSI is formulated. Since the transmit power of each hop is independent of the channel variation of other hops, the power-minimization problem can be decomposed into two levels of optimization [27]:

1) Subproblems: Optimize $P_{t i}$ at different channel-fading states with a given $\bar{p}_{e, i}=p_{\text {req }, i}$ to minimize the average power consumption of the $i$ th hop for $i=1,2, \ldots, N$. For each $i$, this is a classical power-control problem for a one-hop link, which can be written as

$$
\begin{aligned}
\min _{P_{t i}} & \bar{P}_{t i}=\int_{0}^{+\infty} P_{t i} f\left(\alpha_{i}\right) d \alpha_{i} \\
\text { s.t. } & \bar{p}_{e, i}=p_{\text {req }, i} \\
& P_{t i} \geq 0 .
\end{aligned}
$$

According to the KKT necessary optimality condition, the optimal solution of (27), i.e., $P_{t i}^{* *}$, satisfies $P_{t i}^{* *}=0$, or

$$
p_{e}^{\prime}\left(\tilde{\gamma}_{i} P_{t i}^{* *}\right)=-\frac{1}{u_{i} \tilde{\gamma}_{i}}, \quad P_{t i}^{* *}>0
$$

where $u_{i}$ is a positive constant satisfying [23]

$$
\int_{0}^{+\infty} p_{e}\left(\tilde{\gamma}_{i} P_{t i}^{* *}\right) f\left(\alpha_{i}\right) d \alpha_{i}=p_{\text {req }, i} .
$$

Through the preceding two equations, we can obtain $P_{t i}^{* *}$ as a function of $\tilde{\gamma}_{i}$ and $p_{\text {req }, i}$.

2) Master problem: Optimize the allocation of $p_{\text {req }, i}, i=$ $1,2, \ldots, N$ for all hops to minimize the overall power consumption and satisfy the end-to-end BER constraint. This is a power-allocation problem and can be formulated into

$$
\begin{aligned}
\min _{\left\{p_{\text {req }, i}\right\}} & \sum_{i=1}^{N} \bar{P}_{t i}^{* *} \\
& 1-\prod_{i=1}^{N}\left[1-2 p_{\text {req }, i}\right] \\
\text { s.t. } & \frac{2}{2} \leq p_{\text {req }} \\
& 0 \leq p_{\text {req }, i} \leq 1, \text { for } i=1,2, \ldots, N
\end{aligned}
$$


where $\bar{P}_{t i}^{* *}$ is the minimum average power consumption of the $i$ th hop with a given $p_{\text {req }, i}$, which is obtained through the $i$ th subproblem.

Since the target end-to-end BER $p_{\text {req }}$ is very small in general, we can approximate the BER constraint in (30) as in [7]

$$
\sum_{i=1}^{N} p_{\text {req }, i} \leq p_{\text {req }}
$$

It is noted that this approximation makes the BER constraint more strict, and the solution satisfying (31) will also satisfy the original BER constraint. With the approximation, the KKT necessary optimality condition of the master problem is

$$
\frac{\partial \bar{P}_{t i}^{* *}}{\partial p_{\text {req }, i}}+v=0
$$

where $v$ is a positive constant satisfying

$$
\sum_{i=1}^{N} p_{\text {req }, i}=p_{\text {req }}
$$

By combining (28), (29), (32), and (33), we can obtain the optimum solution $P_{t i}^{* *}$ as a function of $\tilde{\gamma}_{i}$.

\section{B. MQAM or MPSK Modulations}

If MQAM or MPSK is used and the BER approximation in (11) is applied, according to (28) and (29), the optimal transmit power of the $i$ th hop is

$$
P_{t i}^{* *}= \begin{cases}\frac{1}{b \tilde{\gamma}_{i}} \ln \left(\tilde{u}_{i} \tilde{\gamma}_{i}\right), & \tilde{u}_{i} \tilde{\gamma}_{i} \geq 1 \\ 0, & \text { otherwise }\end{cases}
$$

where $\tilde{u}_{i}=a b u_{i}$, which is a function of $p_{\text {req }, i}$, and satisfies

$$
p_{\text {req }, i}-\bar{p}_{e, i}=\frac{1-e^{-\frac{N_{0}}{G_{i} \tilde{u}_{i}}}}{2}+\frac{a N_{0}}{G_{i} \tilde{u}_{i}} E_{1}\left(\frac{N_{0}}{G_{i} \tilde{u}_{i}}\right)
$$

where $E_{1}(t)$ is the exponential integral function defined as $E_{1}(t)=\int_{t}^{+\infty}\left(e^{-x} / x\right) d x[24]$.

The minimum average power consumption of the $i$ th hop is thus

$$
\begin{aligned}
\bar{P}_{t i}^{* *} & =\int_{0}^{+\infty} P_{t i}^{* *} f\left(\alpha_{i}\right) d \alpha_{i} \\
& =\int_{1}^{+\infty} \frac{N_{0}}{G_{i} b x} e^{-\frac{N_{0} x}{G_{i} \bar{u}_{i}}} \ln x d x .
\end{aligned}
$$

Inserting (35) and (36) into (32), we obtain

$$
\frac{\tilde{u}_{i}}{\tilde{v}}=1+\frac{\frac{1}{2}-a}{a} \frac{e^{-\frac{N_{0}}{G_{i} \tilde{u}_{i}}}}{E_{1}\left(\frac{N_{0}}{G_{i} \tilde{u}_{i}}\right)}
$$

where $\tilde{v}=a b v$. According to (35), we have $\left(N_{0} / G_{i} \tilde{u}_{i}\right)<$ $p_{\text {req, } i} \ll 1$. Therefore, the second term on the right side of (37) is much smaller than 1. By neglecting this small term, we approximate $\tilde{u}_{i} \approx \tilde{v}$ for $i \in[1, N]$.
An approximation of the optimal power allocation is thus obtained as

$$
\tilde{P}_{t i}^{* *}= \begin{cases}\frac{1}{b \tilde{\gamma}_{i}} \ln \left(\tilde{v} \tilde{\gamma}_{i}\right), & \tilde{v} \tilde{\gamma}_{i} \geq 1 \\ 0, & \text { otherwise }\end{cases}
$$

where $\tilde{v}$ is a positive constant satisfying (33), which is rewritten as

$$
\sum_{i=1}^{N}\left[\frac{1-e^{-\frac{N_{0}}{G_{i} \tilde{v}}}}{2}+a \frac{N_{0}}{G_{i} \tilde{v}} E_{1}\left(\frac{N_{0}}{G_{i} \tilde{v}}\right)\right]=p_{\text {req }} .
$$

It is found that the power allocated for each hop in (38) has the same expression as the power allocation for a single-hop link [16], except that each hop has an identical constant $\tilde{v}$ as a function of all hops' ACSI instead of a unique constant related only to its own ACSI. By substituting $\tilde{v}=\tilde{u}_{i}$ into (35), one can see that, by choosing the same $u_{i}$ for different hops, a smaller target BER $p_{\text {req }, i}$ is allocated to a hop with a larger average channel gain, which makes the power allocation more efficient and results in an overall power saving.

Since $\left(N_{0} / G \tilde{v}\right) \ll 1$, we approximate $e^{-\left(N_{0} / G_{i} \tilde{v}\right)} \approx 1-$ $\left(N_{0} / G_{i} \tilde{v}\right)$ and $E_{1}\left(N_{0} / G_{i} \tilde{v}\right) \approx-\gamma-\ln \left(N_{0} / G_{i} \tilde{v}\right)$ in (39) and obtain [28]

$$
\tilde{v} \approx-N_{0} \frac{l_{1}}{p_{\text {req }}} W_{-1}\left(-\frac{p_{\text {req }} e^{-l_{2} / l_{1}}}{l_{1}}\right)=2 a \tilde{\lambda}
$$

where $\quad l_{1}=a \sum_{i=1}^{N}\left(1 / G_{i}\right)=a k_{1}, \quad$ and $\quad l_{2}=$ $\sum_{i=1}^{N}\left(1 / G_{i}\right)\left((1 / 2)-a \gamma-a \ln \left(1 / G_{i}\right)\right)=a\left(k_{2}-k_{1} \ln a\right) \cdot \gamma$ is the Euler-Mascheroni constant.

Therefore, the power allocation in (38) can be rewritten as

$$
\tilde{P}_{t i}^{* *}= \begin{cases}\frac{1}{b \tilde{\gamma}_{i}} \ln \left(2 a \tilde{\lambda} \tilde{\gamma}_{i}\right), & 2 a \tilde{\lambda} \tilde{\gamma}_{i} \geq 1 \\ 0, & \text { otherwise. }\end{cases}
$$

Similar to $\tilde{P}_{t i}^{*}$ in (21), $P_{t i}^{* *}$ is also linearly increased with the noise power $N_{0}$. Through a comparison of (21) and (41), it is seen that $\tilde{P}_{t i}^{* *}$ can be regarded as an approximation of $\tilde{P}_{t i}^{*}$ by assuming $1-\sum_{k=1, k \neq i}^{N}\left(1 / \tilde{\lambda} \tilde{\gamma}_{k}\right) \approx 1$. This indicates that $P_{t i}^{* *}$ is only slightly larger than $\tilde{P}_{t i}^{*}$. Therefore, the power consumption in the ICSI/ACSI scenario is expected to be larger than but close to the power consumption in the ICSI/ICSI scenario. A numerical comparison will be presented in Section V.

\section{Numerical Results}

Numerical examples are given in this section to illustrate the optimization results. In the wireless channel model, we assume that the reference power gain factor at $d=1 \mathrm{~m}$ is $G_{0}=70 \mathrm{~dB}$ and that the path loss factor $n=3.5$. Since the power allocations are always linearly increased with the noise power, without loss of generality, the noise power is set to $N_{0}=1$. In each communication hop, information bits are 4-QAM modulated, and the required end-to-end BER is set to $p_{\text {req }}=10^{-3}$. The parameters in the BER approximation (11) are $a=0.2$ and $b=1.6 / 3$ [16].

To show the effectiveness of the proposed power-adaptation schemes for multihop links, we compare them with a distributed 


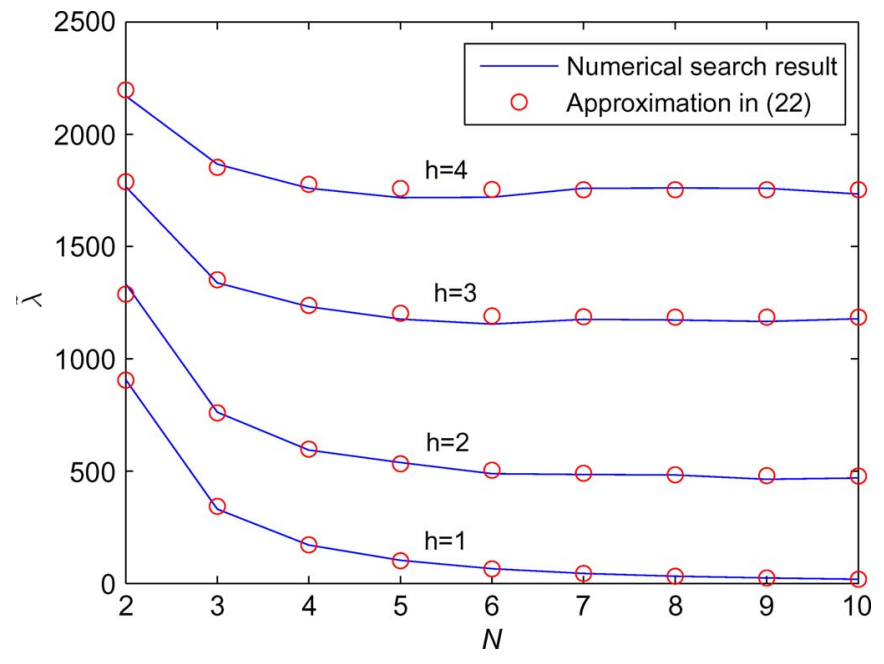

Fig. 1. Comparison of $\tilde{\lambda}$ obtained through numerical search and approximation in (22).

power-adaptation scheme (with an individual-hop BER requirement), which treats each hop as an independent link and adjusts its transmit power by using the conventional single-hop poweradaptation scheme [16]. For an $N$-hop network, the average BER requirement for each individual hop in the distributed scheme is set to $\hat{p}_{\text {req }}=\left(1-\left(1-2 p_{\text {req }}\right)^{(1 / N)}\right) / 2$ to achieve the same end-to-end BER as the proposed scheme.

For a simple illustration of the relationship between the power consumption and the relay nodes' locations, we first consider a linear multihop network model and assume that the proportion between the distances of two adjacent hops is constant, i.e., $r_{2} / r_{1}=r_{3} / r_{2}=\cdots=r_{N} / r_{N-1}=h$, where $h$ is a constant. The distance from the source node to the destination node, i.e., the sum of all hops' communication distances, is set to $100 \mathrm{~m}$.

To validate the approximation of $\tilde{\lambda}$ in (22), Fig. 1 shows a comparison of the values of $\tilde{\lambda}$ obtained through numerical search and the approximation for different hop numbers $N$ and distance proportion $h$. It is seen that (22) approximates the value of $\tilde{\lambda}$ very well.

Figs. 2 and 3 show the power consumptions, with different $h$ 's, obtained through three schemes for two- and three-hop networks, respectively. For the two-hop case in Fig. 2, the power consumptions of the proposed optimization scheme in the ICSI/ICSI scenario using accurate [see (17)] and approximated $\rho$ [see (20)] are both plotted. It is seen that the use of the approximation of $\rho$ does not noticeably impact the average power saving performance.

According to the comparisons in Figs. 2 and 3, the power consumption of the proposed scheme with ICSI/ACSI is very close to, although larger than, the proposed scheme with ICSI/ICSI. This is a very important observation, and it indicates that having the knowledge of other hops' ICSI at each node is not necessary for power adaptation in multihop links. In comparison with the conventional distributed power adaptation scheme, when the communication distance of each hop is not equally distributed, i.e., $h$ is not equal to 1 , the proposed two optimization schemes significantly save the power consumption.

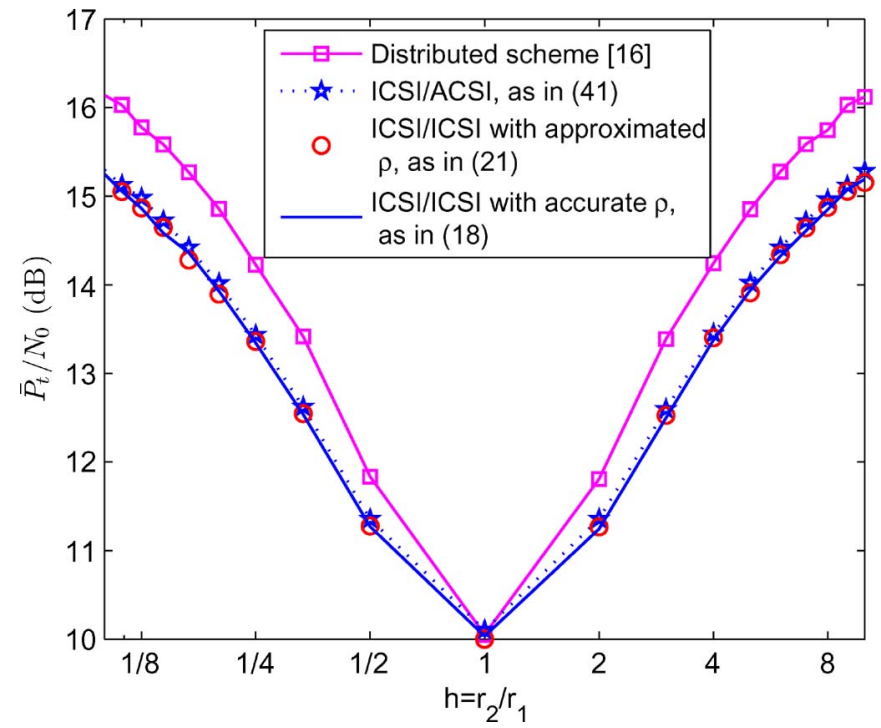

Fig. 2. Power consumptions of the two proposed power-adaptation schemes (with an end-to-end BER requirement) and the distributed power-adaptation scheme (with an individual-hop BER requirement) in a two-hop linear network.

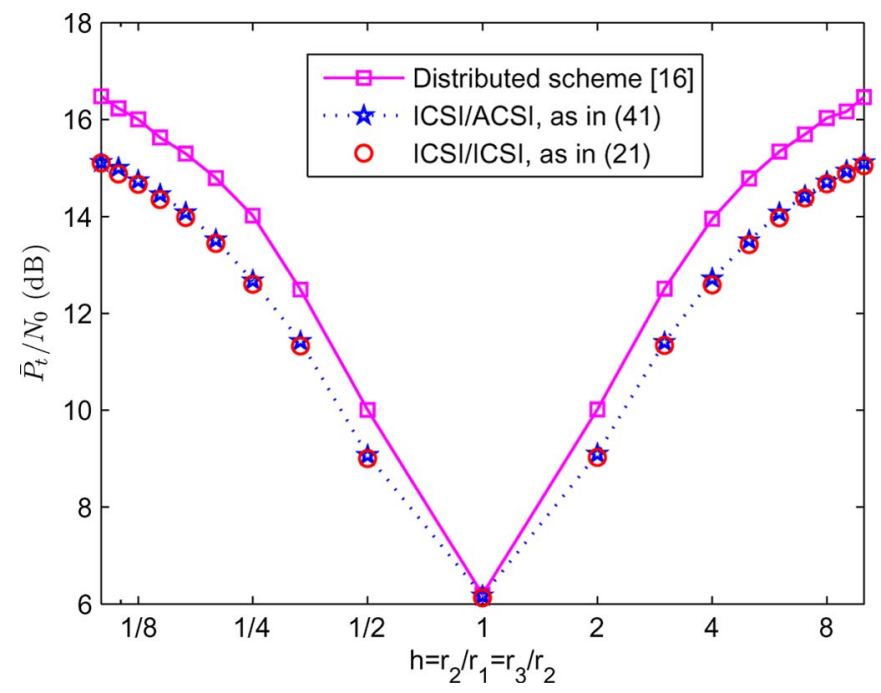

Fig. 3. Power consumptions of the two proposed power-adaptation schemes (with an end-to-end BER requirement) and the distributed power-adaptation scheme (with an individual-hop BER requirement) in a three-hop linear network.

Furthermore, a 2-D randomly deployed multihop network is considered for the comparison of the three power-adaptation schemes, as shown in Fig. 4. We consider an ad hoc network where the nodes are spatially distributed according to a 2-D Poisson distribution with a node density $\lambda_{\text {node }}$ (i.e., the average number of nodes per unit area) [29]. Assume that a simple nearest-neighboring routing scheme is used, i.e., the next-hop node is always the nearest neighbor of the current node within the direction $\pm(\varphi / 2)$ to the axis, as shown in Fig. 4. The distance of each hop is thus Rayleigh distributed with a pdf in [30]

$$
p_{r}(r)=\lambda_{\text {node }} r \varphi e^{-\frac{\lambda_{\text {node }} r^{2} \varphi}{2}} .
$$

By setting $\lambda_{\text {node }}=10^{-3}$ nodes $/ \mathrm{m}^{2}$ and $\varphi=\pi / 6$, we numerically obtain the average power consumptions of the three 


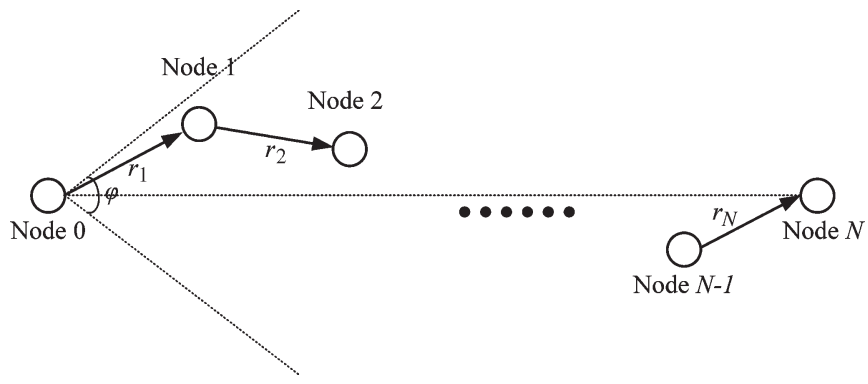

Fig. 4. Two-dimensional randomly deployed multihop network $\left(r_{i}, i=\right.$ $1,2, \ldots, N$ is the communication distance of the $n$th hop).

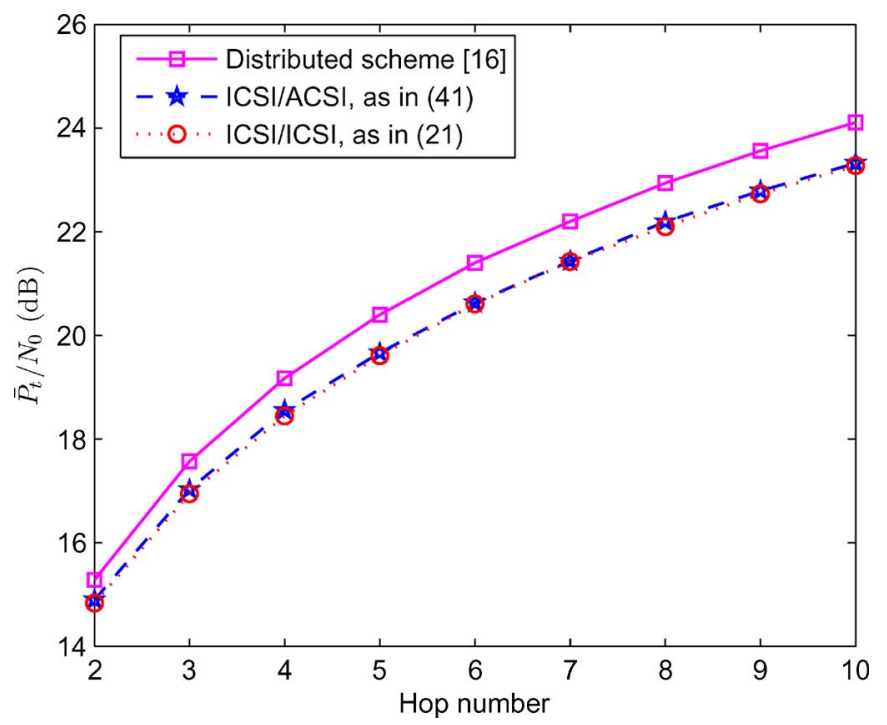

Fig. 5. Comparison of the two proposed power-adaptation schemes (with an end-to-end BER requirement) and the distributed power-adaptation scheme (with an individual-hop BER requirement) in a randomly deployed multihop network.

power-adaptation schemes with different hop numbers in the randomly deployed network, as shown in Fig. 5. The results further validate that the power-adaptation scheme with ICSI/ACSI performs similarly as the power-adaptation scheme with ICSI/ICSI for any number of hops. The power saving of the two proposed schemes over the distributed scheme is increased with the hop number, and its typical value is $0.5-1 \mathrm{~dB}$.

In Fig. 6, we illustrate the average power consumptions of the three schemes, considering the extra power consumption to obtain the ICSI. The data block length is set to $L=1000$ bits, and the number of bits to exchange ICSI is $K=8$ bits. To count in the extra power consumption to obtain the ICSI, the power consumption with ICSI/ICSI is adjusted according to (23), and the power consumption with ICSI/ACSI and the distributed scheme are both adjusted according to (24). It is seen that the power adaptation scheme with ICSI/ICSI obtains increasingly less power savings with the increase in $N$ and even consumes more power when $N>6$, in comparison with the distributed scheme. However, the power-adaptation scheme with ICSI/ACSI maintains its power saving over the distributed scheme for any hop number. The results highlight the impact of the extra power consumption to obtain the ICSI on the performance of power-adaptation schemes. In the ICSI/ICSI

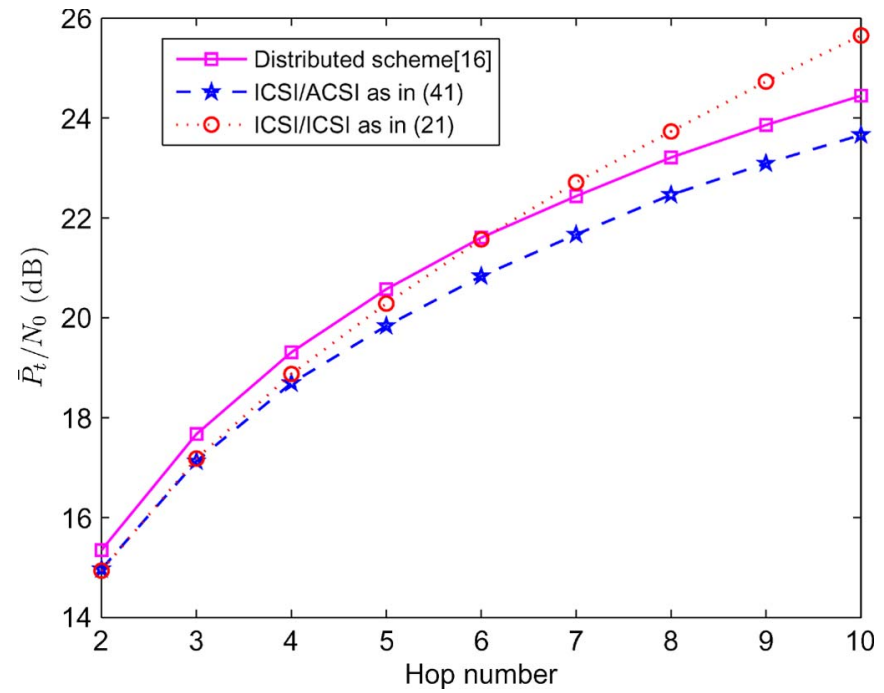

Fig. 6. Comparison of the two proposed power-adaptation schemes (with an end-to-end BER requirement) and the distributed power-adaptation scheme (with an individual-hop BER requirement) in a randomly deployed multihop network considering the extra power to obtain ICSI.

scenario, the ICSI of each hop has to be broadcasted to all the nodes. While in the ICSI/ACSI scenario and the distributed scheme, the ICSI of each hop only needs to be sent back to its own transmitter. Therefore, when the hop number increases, the extra power consumption with ICSI/ICSI will increase much faster than that with ICSI/ACSI or the distributed scheme.

\section{CONCLUSION}

In this paper, the power adaption for multihop networks with the objective of average power minimization and the constraint of an average end-to-end BER requirement has been modeled. Two scenarios with regard to the CSI knowledge have been considered, and the corresponding optimal transmission powers have approximately been derived. Numerical results have been presented to show the power savings through the proposed power-adaptation schemes, compared with the conventional distributed power-adaptation scheme with an individual-hop BER constraint.

\section{APPENDix A \\ PROOF OF (3)}

Proof: This equation can be proven by the use of mathematical induction as follows:

1) When $N=1$, (3) can simply be verified since $p_{0,1}=p_{1}$.

2) According to (2), if

$$
p_{0, k}=\frac{1-\prod_{i=1}^{k}\left(1-2 p_{i}\right)}{2}
$$

we have

$$
\begin{aligned}
p_{0, k+1} & =\frac{1-\left(1-2 p_{0, k}\right)\left(1-2 p_{k+1}\right)}{2} \\
& =\frac{1-\prod_{i=1}^{k+1}\left(1-2 p_{i}\right)}{2} .
\end{aligned}
$$

Equation (3) is thus proven. 


\section{APPENDIX B}

PROOF OF THEOREM 1

Proof: By defining $p_{s}\left(\gamma_{i}\right)=1-2 p_{e}\left(\gamma_{i}\right)$ and substituting (7) and (8) into (6), the power-minimization problem is rewritten as

$$
\begin{array}{ll}
\min _{\left\{P_{t i}\right\}} & \int_{\mathcal{A} \in R_{+}^{N}}\left(\sum_{i=1}^{N} P_{t i}(\tilde{\boldsymbol{\Gamma}})\right) \tilde{f}(\mathcal{A}) d \mathcal{A} \\
\text { s.t. } & \int_{\mathcal{A} \in R_{+}^{N}} \frac{1-\prod_{i=1}^{N} p_{s}\left(\tilde{\gamma}_{i} P_{t i}(\tilde{\boldsymbol{\Gamma}})\right)}{2} \tilde{f}(\mathcal{A}) d \mathcal{A} \leq p_{\text {req }} \\
& P_{t i}(\tilde{\boldsymbol{\Gamma}}) \geq 0, \text { for } \forall \tilde{\boldsymbol{\Gamma}} \text { and } i=1,2, \ldots, N .
\end{array}
$$

The KKT necessary conditions for the optimal solution $P_{t}^{*}(\tilde{\boldsymbol{\Gamma}})=\left(P_{t 1}^{*}(\tilde{\boldsymbol{\Gamma}}), P_{t 2}^{*}(\tilde{\boldsymbol{\Gamma}}), \ldots, P_{t N}^{*}(\tilde{\boldsymbol{\Gamma}})\right)$ are [16], [23]

$$
\begin{aligned}
& 1-\frac{1}{2} \lambda \tilde{\gamma}_{i} p_{s}^{\prime}\left(\tilde{\gamma}_{i} P_{t i}^{*}(\tilde{\boldsymbol{\Gamma}})\right) \prod_{\substack{k=1 \\
k \neq i}}^{N} p_{s}\left(\tilde{\gamma}_{k} P_{t k}^{*}(\tilde{\boldsymbol{\Gamma}})\right)-\mu_{i}(\tilde{\boldsymbol{\Gamma}})=0 \\
& \mu_{i}(\tilde{\boldsymbol{\Gamma}}) P_{t i}^{*}(\tilde{\boldsymbol{\Gamma}})=0 \\
& \mu_{i}(\tilde{\boldsymbol{\Gamma}}) \geq 0 \\
& P_{t i}^{*}(\tilde{\boldsymbol{\Gamma}}) \geq 0 \\
& \lambda \geq 0
\end{aligned}
$$

for $\forall \tilde{\boldsymbol{\Gamma}} \in R_{+}^{N}$, and $\forall i \in[1, N]$, and

$\lambda\left\{1-2 p_{\text {req }}-\int_{\mathcal{A} \in R_{+}^{N}} \prod_{i=1}^{N} p_{s}\left(\tilde{\gamma}_{i} P_{t i}^{*} P_{t i}^{*}(\tilde{\boldsymbol{\Gamma}})\right) \tilde{f}(\mathcal{A}) d \mathcal{A}\right\}=0$.

According to (44)-(48), for $\forall \tilde{\boldsymbol{\Gamma}} \in R_{+}^{N}$ and $\forall i \in[1, N]$, at least one of the following two conditions must be satisfied: 1) $P_{t i}^{*}(\tilde{\boldsymbol{\Gamma}})=0$ and 2) $\mu_{i}(\tilde{\boldsymbol{\Gamma}})=0$ and $\lambda>0$. If condition 1 is satisfied, $P_{t i}^{*}(\tilde{\boldsymbol{\Gamma}})=0$. If condition 2 is satisfied, by inserting $\mu_{i}(\tilde{\boldsymbol{\Gamma}})=0$ into (44) and $\lambda>0$ into (49), we obtain

$$
\begin{aligned}
\frac{p_{s}\left(\tilde{\gamma}_{i} P_{t i}^{*}(\tilde{\boldsymbol{\Gamma}})\right)}{p_{s}^{\prime}\left(\tilde{\gamma}_{i} P_{t i}^{*}(\tilde{\boldsymbol{\Gamma}})\right)} & =\frac{1}{2} \lambda \tilde{\gamma}_{i} \rho(\tilde{\boldsymbol{\Gamma}}) \\
\int_{\mathcal{A} \in R_{+}^{N}} \rho(\tilde{\boldsymbol{\Gamma}}) \tilde{f}(\mathcal{A}) d \mathcal{A} & =1-2 p_{\text {req }}
\end{aligned}
$$

where $\rho(\tilde{\boldsymbol{\Gamma}})=\prod_{i=1}^{N} p_{s}\left(\tilde{\gamma}_{i} P_{t i}^{*}(\tilde{\boldsymbol{\Gamma}})\right)$. Therefore, the possible value of $P_{t i}^{*}(\tilde{\boldsymbol{\Gamma}})$ is 0 or a positive number that satisfies (50) and (51). Theorem 1 is thus proven.

\section{APPENDIX C}

\section{APPROXIMATION OF $\tilde{\lambda}$}

According to (10) and (20), $\tilde{\lambda}$ is a solution of

$$
g(\tilde{\lambda})=\int_{\sum_{i=1}^{N} \frac{1}{\tilde{\gamma}_{i} \bar{\lambda}} \leq 1-\rho_{\min }}\left(1-\sum_{i=1}^{N} \frac{1}{\tilde{\gamma}_{i} \tilde{\lambda}}\right) \tilde{f}(\mathcal{A}) d \mathcal{A}=1-2 p_{\text {req }} .
$$

With the definition of $\rho_{\min }=((1 / 2 a)-1)\left(1 / \tilde{\lambda} \tilde{\gamma}_{\min }\right)$, the integral region in the preceding equation is rewritten as

$$
\frac{1}{\tilde{\gamma}_{\min } \tilde{\lambda}} \leq 2 a\left(1-\sum_{i=1, i \neq i_{0}}^{N} \frac{1}{\tilde{\gamma}_{i} \tilde{\lambda}}\right)
$$

where $i_{0}=\arg \min _{i}\left\{\gamma_{i}\right\}$.

Since $p_{\text {req }} \ll 1, \sum_{i=1}^{N}\left(1 / \tilde{\gamma}_{i} \tilde{\lambda}\right)$ should be much less than 1 under most fading conditions. Therefore, we approximate the aforementioned integral region by $\left(1 / \tilde{\gamma}_{\min } \tilde{\lambda}\right) \leq 2 a$ and derive the approximation of $g(\tilde{\lambda})$ as

$$
\begin{aligned}
g(\tilde{\lambda}) & \approx \int_{\frac{1}{\tilde{\gamma}_{\min } \bar{\lambda}} \leq 2 a}\left(1-\sum_{i=1}^{N} \frac{1}{\tilde{\gamma}_{i} \tilde{\lambda}}\right) \tilde{f}(\mathcal{A}) d \mathcal{A} \\
& =\int_{\frac{1}{\tilde{\gamma}_{1} \bar{\lambda}} \leq 2 a} \ldots \int_{\frac{1}{\tilde{\gamma}_{N} \lambda} \leq 2 a}\left(1-\sum_{i=1}^{N} \frac{1}{\tilde{\gamma}_{i} \tilde{\lambda}}\right) \tilde{f}(\mathcal{A}) d \mathcal{A} \\
& =e^{-\sum_{i=1}^{N} \frac{N_{0}}{2 a G_{i} \bar{\lambda}}}-\sum_{i=1}^{N} \frac{N_{0} E_{1}\left(\frac{N_{0}}{2 a \tilde{\lambda} G_{i}}\right)}{\tilde{\lambda} G_{i}} e^{-\sum_{\substack{k=1 \\
k \neq i}}^{N} \frac{N_{0}}{2 a G_{k} \lambda}} .
\end{aligned}
$$

By substituting (54) into (52), we can obtain that $\sum_{i=1}^{N}\left(N_{0} / 2 a G_{i} \tilde{\lambda}\right)<2 p_{\text {req }} \ll 1$. Therefore, we approximate $g(\hat{\lambda})$ using the Taylor expansion $e^{-x} \approx 1-x$ and $E_{1}(x) \approx$ $-\gamma-\ln x$ as [28]

$$
g(\tilde{\lambda}) \approx 1-A_{1}-A_{2}+A_{3}
$$

where

$$
\begin{aligned}
A_{1} & =\sum_{i=1}^{N} \frac{N_{0}}{2 a G_{i} \tilde{\lambda}} \\
A_{2} & =\sum_{i=1}^{N} \frac{N_{0}}{\tilde{\lambda} G_{i}}\left(-\gamma-\ln \frac{N_{0}}{2 a \tilde{\lambda} G_{i}}\right) \\
A_{3} & =\sum_{i=1}^{N} \frac{N_{0}}{\tilde{\lambda} G_{i}}\left(-\gamma-\ln \frac{N_{0}}{2 a \tilde{\lambda} G_{i}}\right) \sum_{k=1, k \neq i}^{N} \frac{N_{0}}{2 a G_{k} \tilde{\lambda}} .
\end{aligned}
$$

Since $A_{1} \ll 1, A_{3}<A_{1} A_{2}$, and $A_{1}+A_{2}-A_{3}=2 p_{\text {req }}$ according to (52), it is easy to obtain that $A_{2} \ll 1, A_{3} \ll A_{1}$, and $A_{3} \ll A_{2}$. Therefore, we neglect $A_{3}$ and approximate

$$
g(\tilde{\lambda}) \approx 1-\sum_{i=1}^{N} \frac{N_{0}}{2 a G_{i} \tilde{\lambda}}-\sum_{i=1}^{N} \frac{N_{0}}{\tilde{\lambda} G_{i}}\left(-\gamma-\ln \frac{N_{0}}{2 a \tilde{\lambda} G_{i}}\right) .
$$

By substituting (59) into (52), we obtain that

$$
2 p_{\text {req }} \frac{\tilde{\lambda}}{N_{0}}-k_{1} \ln \frac{\tilde{\lambda}}{N_{0}}-k_{2} \approx 0
$$

where $k_{1}=\sum_{i=1}^{N}\left(1 / G_{i}\right)$, and $k_{2}=\sum_{i=1}^{N}\left(1 / G_{i}\right)((1 / 2 a)-$ $\left.\gamma-\ln \left(1 / 2 a G_{i}\right)\right)$. Since $\sum_{i=1}^{N}\left(N_{0} / G_{i} \tilde{\lambda}\right)<2 p_{\text {req }}$ according to 
(52) and (54), we have

$$
\tilde{\lambda} \approx-N_{0} \frac{k_{1}}{2 p_{\text {req }}} W_{-1}\left(-\frac{2 p_{\text {req }} e^{-k_{2} / k_{1}}}{k_{1}}\right)
$$

where $W_{-1}(x)$ is the branch of the Lambert $\mathrm{W}$ function that satisfies $W_{-1}(x) \leq-1$. The Lambert $\mathrm{W}$ function is defined as the multivalued inverse of the function $f(w)=w e^{w}$ [25].

\section{REFERENCES}

[1] S. Cui, A. Goldsmith, and A. Bahai, "Energy-constrained modulation optimization," IEEE Trans. Wireless Commun., vol. 4, no. 5, pp. 23492360, Sep. 2005.

[2] I. Demirkol, C. Ersoy, and F. Alagöz, "MAC protocols for wireless sensor networks: A survey," IEEE Commun. Mag., vol. 44, no. 4, pp. 115-121, Apr. 2006.

[3] W. R. Heinzelman, J. Kulik, and H. Balakrishnan, "Adaptive protocols for information dissemination in wireless sensor networks," in Proc. 5th Annu. ACM/IEEE Int. Conf. Mobile Comput. Netw., Seattle, WA, Aug. 1999, pp. 174-185.

[4] R. Madan, S. Cui, S. Lall, and A. Goldsmith, "Modeling and optimization of transmission schemes in energy-constrained wireless sensor networks," IEEE/ACM Trans. Netw., vol. 15, no. 6, pp. 1359-1372, Dec. 2007.

[5] H. Cheng, Y.-D. Yao, and S. Quoraishee, "Optimization of energy consumption in sensor networks," in Proc. 26th Army Sci. Conf., Orlando, FL, Dec. 2008.

[6] L. Piazzo, "A practical algorithm for power minimisation in wireless networks by means of multi-hop and load partitioning," IEEE Trans. Commun., vol. 56, no. 2, pp. 270-278, Feb. 2008.

[7] A. P. T. Lau and S. Cui, "Joint power minimization in wireless relay channels," IEEE Trans. Wireless Commun., vol. 6, no. 8, pp. 2820-2824, Aug. 2007.

[8] T. Cover and A. Gamal, "Capacity theorems for the relay channel," IEEE Trans. Inf. Theory, vol. IT-25, no. 5, pp. 572-584, Sep. 1979.

[9] J. Laneman, D. Tse, and G. Wornell, "Cooperative diversity in wireless networks: Efficient protocols and outage behavior," IEEE Trans. Inf. Theory, vol. 50, no. 12, pp. 3062-3080, Dec. 2004.

[10] N. Ahmed, M. Khojastepour, A. Sabharwal, and B. Aazhang, "Outage minimization with limited feedback for the fading relay channel," IEEE Trans. Commun., vol. 54, no. 4, pp. 659-669, Apr. 2006.

[11] A. Høst-Madsen and J. Zhang, "Capacity bounds and power allocation for wireless relay channels," IEEE Trans. Inf. Theory, vol. 51, no. 6, pp. 2020-2040, Jun. 2005.

[12] M. Hasna and M.-S. Alouini, "Optimal power allocation for relayed transmissions over Rayleigh-fading channels," IEEE Trans. Wireless Commun., vol. 3, no. 6, pp. 1999-2004, Nov. 2004.

[13] Y. Li, B. Vucetic, Z. Zhou, and M. Dohler, "Distributed adaptive power allocation for wireless relay networks," IEEE Trans. Wireless Commun., vol. 6, no. 3, pp. 948-958, Mar. 2007.

[14] J. Hayes, "Adaptive feedback communications," IEEE Trans. Commun. Technol., vol. COM-16, no. 1, pp. 29-34, Feb. 1968.

[15] Y. H. Lee and Y. Bar-Ness, "Power adaptation for BPSK signaling with average and peak power constraints in Rayleigh fading channels," IEEE Trans. Commun., vol. 51, no. 11, pp. 1871-1876, Nov. 2003.

[16] S. T. Chung and A. Goldsmith, "Degrees of freedom in adaptive modulation: A unified view," IEEE Trans. Commun., vol. 49, no. 9, pp. 15611571, Sep. 2001

[17] J. Boyer, D. Falconer, and H. Yanikomeroglu, "Multihop diversity in wireless relaying channels," IEEE Trans. Commun., vol. 52, no. 10, pp. 18201830 , Oct. 2004

[18] X. Zhang and Y. Gong, "Adaptive power allocation for multihop regenerative relaying with limited feedback," IEEE Trans. Veh. Technol., vol. 58, no. 7, pp. 3862-3867, Sep. 2009.

[19] A. Aduwo and A. Annamalai, "Channel-aware inter-cluster routing protocol for wireless ad-hoc networks exploiting network diversity," in Proc. IEEE 60th VTC-Fall, Sep. 2004, vol. 4, pp. 2858-2862.

[20] E. Biglieri, J. Proakis, and S. Shamai, "Fading channels: Informationtheoretic and communications aspects," IEEE Trans. Inf. Theory, vol. 44, no. 6, pp. 2619-2692, Oct. 1998.

[21] A. Muller and J. Speidel, "Achieving exponential diversity in wireless multihop systems with regenerative relays," in Proc. IEEE ICC, Jun. 2009, pp. 1-6.
[22] V. Sharma, K. Premkumar, and R. Swamy, "Exponential diversity achieving spatio-temporal power allocation scheme for fading channels," IEEE Trans. Inf. Theory, vol. 54, no. 1, pp. 188-208, Jan. 2008.

[23] M. S. Bazaraa, H. D. Sherali, and C. M. Shetty, Nonlinear Programming: Theory and Algorithms., 3rd ed. Hoboken, NJ: Wiley, 2006.

[24] I. S. Gradshtekyn and I. M. Ryzhik, Table of Integrals, Series, and Products. San Diego, CA: Academic, 1980.

[25] R. M. Corless, G. H. Gonnet, D. E. G. Hare, D. J. Jeffrey, and D. E. Knuth, "On the Lambert W function," Adv. Comput. Math., vol. 5, pp. 329-359, 1996.

[26] R. Madan, N. Mehta, A. Molisch, and J. Zhang, "Energy-efficient cooperative relaying over fading channels with simple relay selection," IEEE Trans. Wireless Commun., vol. 7, no. 8, pp. 3013-3025, Aug. 2008.

[27] D. Palomar and M. Chiang, "A tutorial on decomposition methods for network utility maximization," IEEE J. Sel. Areas Commun., vol. 24, no. 8, pp. 1439-1451, Aug. 2006.

[28] N. Bleistein and R. A. Handelsman, Asymptotic Expansions of Integrals. New York: Dover, 1986.

[29] E. S. Sousa and J. A. Silvester, "Optimum transmission ranges in a directsequence spread-spectrum multihop packet radio network," IEEE J. Sel. Areas Commun., vol. 8, no. 5, pp. 762-771, Jun. 1990.

[30] M. Haenggi, "On routing in random Rayleigh fading networks," IEEE Trans. Wireless Commun., vol. 4, no. 4, pp. 1553-1562, Jul. 2005.

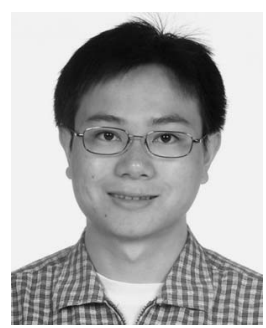

Hongbing Cheng (S'03-M'07) received the B.S. and Ph.D. (with honors) degrees in electronic engineering from Peking University, Beijing, China, in 2002 and 2007, respectively.

$\mathrm{He}$ is currently a Research Assistant Professor with the Wireless Information System Engineering Laboratory, Department of Electrical and Computer Engineering, Stevens Institute of Technology, Hoboken, NJ. His research interests include wireless communication theory, resource allocation for cellular and ad hoc networks, broadband code-division multiple access, beamforming, cooperative relay, and cognitive radio.

Dr. Cheng served as a Technical Session Chair of the 2009 Wireless and Optical Communication Conference and a Technical Program Chair member of the 2010 IEEE International Communications Conference and the 2008 IEEE Global Telecommunications Conference, and was invited as a technical reviewer for many technical journals and conferences.

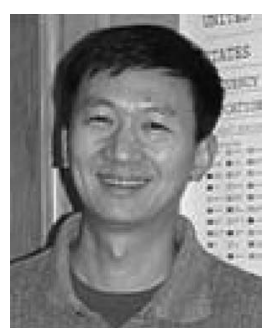

Yu-Dong Yao (S'88-M'88-SM'94) received the B.Eng. and M.Eng. degrees in electrical engineering from Nanjing University of Posts and Telecommunications, Nanjing, China, in 1982 and 1985, respectively, and the Ph.D. degree in electrical engineering from Southeast University, Nanjing, China, in 1988.

From 1989 and 1990, he was with Carleton University, Ottawa, ON, Canada, as a Research Associate, working on mobile radio communications. From 1990 to 1994, he was with Spar Aerospace Ltd., Montreal, QC, Canada, where he was involved in research on satellite communications. From 1994 to 2000, he was with Qualcomm Inc., San Diego, CA, where he participated in research and development of wireless code-division multiple-access (CDMA) systems. Since 2000, he has been with Stevens Institute of Technology, Hoboken, NJ, where he is currently a Professor and the Department Chair of electrical and computer engineering and a Director of the Wireless Information Systems Engineering Laboratory. He is the holder of one Chinese patent and 13 U.S. patents. His research interests include wireless communications and networks, spread spectrum and CDMA, antenna arrays and beamforming, cognitive and software defined radio, and digital signal processing for wireless systems.

Dr. Yao was an Associate Editor for the IEEE COMMUNICATIONS LETTERS and the IEEE TRANSACTIONS ON VEHICULAR TECHNOLOGY and an Editor for the IEEE TRANSACTIONS ON WIRELESS COMMUNICATIONS. 